\title{
Retinal Peripheries Generate Illusory Motion Reversals
}

\author{
Ahmad Yousef ${ }^{1}$ \\ ${ }^{1}$ School of Computational Science and Engineering, McMaster University, Hamilton, Ontario, Canada \\ *Correspondence: mohamas2@mcmaster.ca
}

\begin{abstract}
This article provides evidence that the retinal peripheries generate motion reversal perception. Restricted access of the light rays to retinal peripheries due to pupil constriction is found to significantly limit the perception of motion reversal. We used unfolded stimulus, namely, a linear stimulus instead one a radial one to easily validate our theory. Introduction

Illusory motion reversal happen sporadically while watching continuous smooth motion. This has been theorized as evidence of discrete temporal sampling by the visual system (Purves, etal. 1996; VanRullen, etal., 2005). Other theorists suggested that illusory motion reversal is a product of perceptual rivalry rather than result of discrete sampling, namely, a rivalry between activated and spuriously activated motion detectors (Kline, etal. 2004, 2006). This might be an approach to our recent findings of two integrative conscious brains. Kline and his colleagues continue to provide
\end{abstract} further evidences about their perceptual rivalry theory, namely, the found that illusory reversals of two spatially overlapping and orthogonal motions often

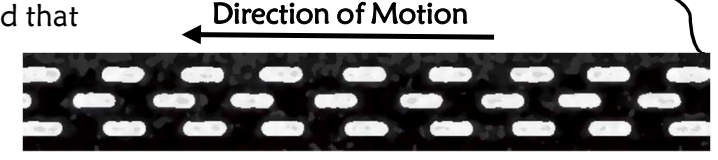
a frame of reference for counterbalancing purposes. The frame of reference is to request the subjects to have central free viewing under maximum brightness. Unlike the frame of reference, all of the experimental attributes must be viewed peripherally.

The first two attributes are to compare the differences between maximum brightness (pupil constriction) and minimum brightness (pupil dilation). The third attribute is about manipulating the contrast, namely, reducing it from 1 to 0.2 . Contrast reduction is known to constrict the pupil and therefore reducing the accessibility to the retinal peripheries (see Wang, 2014). Important reminder, to validate our theory, unlike the frame of reference, all of the attributed stimuli will be viewed peripherally.

Each human subject has to report the number of illusory motion reversals after each trail whether it's from a frame of reference or any of an attributed stimulus.

occur separately. In 2014, Levichkina and her colleagues suggested spatial proximity rather than temporal frequency determines the illusory motion reversals. We, however, think that the illusory motion reversals are perceptually generated by the retinal peripheries, and there is unstoppable rivalry between the perceptions produced by the macula versus the retinal peripheries.

\section{Materials \& Methods}

Twelve human subjects were recruited to participate in this study. The stimulus subtends 12.9 DVA length and 1.37 DVA width and consisted of 9 cycles. The stimulus runs in different speeds, namely, 7.51 degrees/s, 9.13 degrees/s, 10.75 degrees/s, and 12.37 degrees/s. Each human subject will experience twelve trails for each speed, total of 48 trails, and each trail lasts 30 seconds. Three different attributes were tested, all of them manipulate the pupil size. All of them are compared to condition. Human subject will be initially and entirely excluded from performing the experiment, if and only if, he/she see any motion reversals at the aforementioned stage with any of the aforementioned speeds. The data are collected manually from all of the twelve subjects, and then analyzed using the simplest statistical means, namely, the average and the standard error of the mean. In fact, a few subjects had reported motion reversals from the frame of reference condition which came after the attributed stimulus, but the number had never exceeded two reversals. We therefore subtracted these motion reversals to show the effect of the peripheral vision, namely, the contribution of the retinal peripheries on generating illusory motion reversals. Importantly, dim-high contrast value of 1 means the lowest brightness with the greatest contrast, namely absolute white unfolded strip against absolute black background), however, bright- low contrast (0.5) means the greatest brightness 
against gray moving strip that has RGB values half of the absolute white, etc.

Results and Conclusion Significant increase in the number of motion reversals when the stimulus is peripherally viewed regardless the type of the stimulus. As shown in the central infographic, attributes that cause pupil constriction has significant

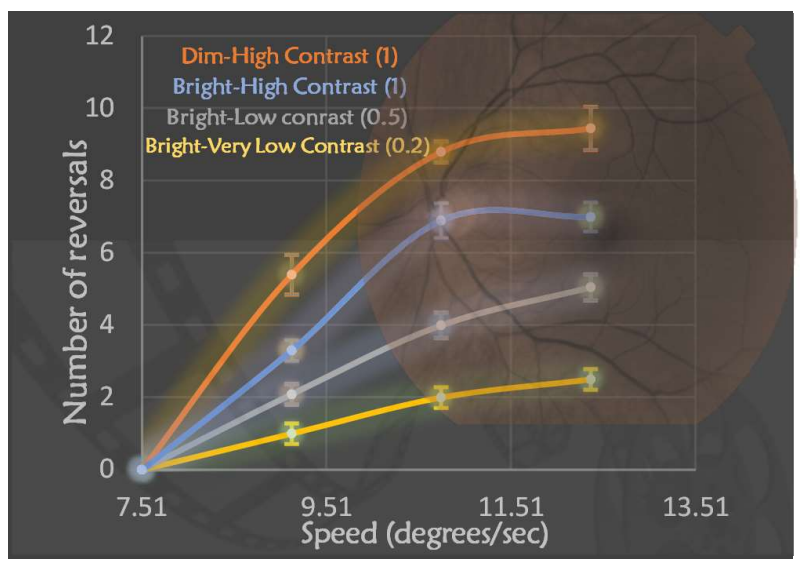

conscious brains, central conscious brain versus peripheral conscious brain (for further information see reference 6,11 , and 12 ). The real challenges in term of neurophysiology, is to figure out the network of the two conscious brains, the central brain, \& the peripheral one. Essential Notification Readers should have direct access to the stimuli, see lower number of motion reversals. Important to remind, each of these attributes were sandwiched by two frames of reference, and each attributed stimulus had been only viewed peripherally. Clearly, the highest brightness constricts the pupil and low contrast adds further constriction to the pupil size, and therefore the lowest accessibility to the retinal peripheries. Astonishingly, the lowest number of motion reversals came out of these attributes (brightvery low contrast value of 0.2 ). High contrast and dim environment, however, dilate the pupil size, astoundingly, the highest number of motion reversal came out of these attributes (dim-high contrast value of 1). Pupil variation has therefore outstanding contribution in the perception of illusory motion reversal. Needless to mention, the pupil dynamics affect the lens dynamics, namely, pupil dilation may be linked with rounded anatomical lens, and thus, additional accessibility to retinal peripheries (see reference 9). All of the twelve subjects had noticed kind of acceleration just before each reversal; but for the speed of 7.51 degrees/s, they noticed only acceleration but not reversals.

Important to mention, the brain do two corrective geometrical transformations of the retinal images, namely, the brain flips the image up from the downside, and then mirror it. It seems like the neurological networks between the retinal peripheries and the visual awareness is unable to do the mirror but the upside-down transformation under certain spatiotemporal conditions, and thus, illusory motion reversals (try the asymmetric stimulus at reference 12 , for better understanding). This might be because the retinal peripheries signal the brain much faster (see reference 10). We, conclusively, strongly agree with Keith Kline and his colleagues prospective about the perceptual rivalry theory, but we would like to be cautious and say, it's a rivalry between two integrative reference 13. Advisably, download the video for the best quality. For validation process, watch all of the videos freely and confirm that there are no illusory motion reversals, and then watch them peripherally. Readers should connect the author directly in case of they cannot access the stimuli. Scholars with brain imaging facilitations are welcome to collaborate in the aforementioned challenging investigations, namely, to localize the spatiotemporal properties of the two conscious brains (central and peripheral). Enquiries should be sent to the author. The stimuli has copyrights, \& they cannot be used for any purpose without a permission form the author. Transactional References

[1] Purves, etal.(1996). The wagon-wheel illusion in movies and reality. PNAS.

[2] VanRullen, etal.(2005). Attention-driven discrete sampling of motion perception. PNAS

[3] Kline, K. Holcombe, A. O. Eagleman, D. M. (2004). Illusory motion reversal is caused by rivalry, not by perceptual snapshots of the visual field. Vision Research, 44, 2653-2658.

[4] Kline, etal. (2006). Illusory motion reversal does not imply discrete processing: Reply to Rojas et al. Vision Research. [5] Keith A. Kline; Eagleman (2008), Evidence against the temporal subsampling account of illusory motion reversal. Journal of Vision.

[6] Ahmad Yousef, etal. 2019. Illusory Fields and Rays. DOI: 10.13140/RG.2.2.29389.49129/3.

[7] Ekaterina Levichkina, Georgy Fedorov, Cees van Leeuwen. Spatial proximity rather than temporal frequency determines the wagon wheel illusion. Perception, 2014.

[8] Wang CA, Munoz DP. (2014) Modulation of stimulus contrast on the human pupil orienting response, in European Journal of Neuroscience, 40(5):2822-32.

[9] Yousef, Ahmad. 2019. "Linking Dynamics Between Pupil and Lens." engrXiv. doi:10.31224/osf.io/9c3vk.

[10] Masland, (2017). Vision: Two Speeds in the Retina.

[11] Yousef, Ahmad. 2019. "Two Distinct Fusiform Face Areas." PsyArXiv. doi:10.31234/osf.io/a8gzv.

[12] Yousef, Ahmad. 2019. "Spatial Attention Dilates Time and Length." PsyArXiv. doi:10.31234/osf.io/qazj9.

[13] The stimuli may be found here: https://drive.google.com/drive/folders/1PsXVUc7a6fKuLjWP4OU AwgiDfakVEC_S?usp=sharing 